Journal of Interconnection Networks

Vol. 9, No. 4 (2008) 507-509

(C) World Scientific Publishing Company

\title{
AUTHOR INDEX \\ VOLUME 9 (2008)
}

Abu-Ghazaleh, N. B., see Kolar, V.

Aikebaier, A., Barolli, V., Enokido, T. and Takizawa, M., A protocol for making an agreement in a peer-to-peer $(\mathrm{p} 2 \mathrm{p})$ society

$\mathbf{9}, 3$ (2008) 205-230

9, 4 (2008) 317-335

Akon, M. M., Goswami, D., Li, H. F., (Sherman) Shen, X. and Singh, A., A novel software-built parallel machines and their interconnections

Al-Mutairi, T. G., see Tayu, S.

Alba, E., see Danoy, G.

Awan, I., see Jing, Z.

Barolli, L., see Durresi, A.

Barolli, V., see Aikebaier, A.

Bataineh, S., Divisible load distribution in a network of processors

Bermond, J.-C., Coudert, D. and Leveque, B., Approximations for all-to-all uniform traffic grooming on unidirectional rings

Bononi, L. and Di Felice, M., Cross-layered mac and multipath routing protocols in multi-hop wireless mobile networks

Boukerche, A., see Samarah, S.

Bouvry, P., see Danoy, G.

Cheng, E., see Lin, C.-K.

Coudert, D., see Bermond, J.-C.

Danoy, G., Alba, E. and Bouvry, P., Optimal interconnection of ad hoc injection networks

Datta, A., see Phua, V.

Di Felice, M., see Bononi, L.

Durresi, A., Durresi, M. and Barolli, L., Priority based wireless communications for health monitoring on highways

Durresi, M., see Durresi, A.

Enokido, T., see Aikebaier, A.

Goswami, D., see Akon, M. M.

Guan, L., see Jing, Z.

Hara, T. and Madria, S. K., Data dissemination methods for time-constraint applications in mobile ad hoc networks

Hart, T. E., see Li, C.

Henry, K. J., see Li, C.

Hongxia, L., see Jing, Z.

Hsu, L.-H., see Lin, C.-K.

Ito, H., see Takabatake, T.

Jia, X., see Lee, J. J.

9, 1 \& 2 (2008) 1-29

9, 1 \& 2 (2008) 141-155

9, 3 (2008) 277-297

9, 4 (2008) 369-387

9, 4 (2008) 337-349

9, 4 (2008) 317-335

9, 1 \& 2 (2008) 31-51

$\mathbf{9}, 4(2008) 471-486$

9, 3 (2008) 177-203

9, 3 (2008) 255-276

9, 3 (2008) 277-297

9, 1 \& 2 (2008) 83-97

9, 4 (2008) 471-486

9, 3 (2008) 277-297

9, 3 (2008) 231-254

9, 3 (2008) 177-203

9, 4 (2008) 337-349

9, 4 (2008) 337-349

9, 4 (2008) 317-335

9, 1 \& 2 (2008) 1-29

9, 4 (2008) 369-387

9, 4 (2008) 351-367

9, 4 (2008) 487-505

9, 4 (2008) 487-505

9, 4 (2008) 369-387

9, 1 \& 2 (2008) 83-97

9, 1 \& 2 (2008) 127-139

9, 1 \& 2 (2008) 73-82 
Jing, Z., Xuefen, C., Guan, L., Hongxia, L. and Awan, I., Design of fuzzy based multi-constrained routing protocol and the performance evaluation

Kesselman, A. and Rosén, A., Controlling CIOQ switches with priority queuing and in multistage interconnection networks

Kolar, V. and Abu-Ghazaleh, N. B., Globally aware routing in multi-hop wireless networks: a formulation and analysis

Landfeldt, B., see Manitpornsut, S.

Lee, J. J., Sheu, E. J. and Jia, X., Extremal Cayley graphs of finite cyclic groups

Leveque, B., see Bermond, J.-C.

Li, C., Hart, T. E., Henry, K. J. and Neufeld, I. A., Average-case "messy" broadcasting

Li, H. F., see Akon, M. M.

Lin, C.-K., Tan, J. J. M., Hsu, L.-H., Cheng, E. and Lipták, L., Conditional diagnosability of Cayley graphs generated by transposition trees under the comparison diagnosis model

Lipták, L., see Lin, C.-K.

Luo, J., see Qu, Y.

Madria, S. K., see Hara, T.

Mahfoudh, S. and Minet, P., Eolsr: an energy efficient routing protocol in wireless ad hoc and sensor networks

Manitpornsut, S. and Landfeldt, B., Weighted channel allocation and power control for self-configurable infrastructure WLANS

Minet, P., see Mahfoudh, S.

Moadeli, M., Vanderbauwhede, W. and Shahrabi, A., Communication modeling of QOS-aware wormhole-routed NOCS

Nakamigawa, T., see Takabatake, T.

Neufeld, I. A., see Li, C.

Parhami, B., Periodically regular chordal rings are preferable to double-ring networks

Phua, V. and Datta, A., A link state dependent TDMA protocol for industrial wireless sensor network applications in periodically changing environments

Qu, Y., Luo, J., Wang, P. and Yang, L. T., A novel spectrum of epoch convergent congestion control

Rosén, A., see Kesselman, A.

Samarah, S. and Boukerche, A., Chronological tree - a compressed structure for mining behavioral patterns from wireless sensor networks

Scherson, I. D., see Valencia, D. S.

Shahrabi, A., see Moadeli, M.

Sheltami, T. R., An efficient neighbor-aware protocol for SNET formation

(Sherman) Shen, X., see Akon, M. M.

Sheu, E. J., see Lee, J. J.

Singh, A., see Akon, M. M.

Srinivasan, B., see Zhao, G.

Takabatake, T., Nakamigawa, T. and Ito, H., Connectivity of generalized hierarchical completely-connected networks

Takizawa, M., see Aikebaier, A. $\mathbf{9}, 4(2008) 369-387$

$\mathbf{9}, 1$ \& $2(2008) 53-72$

$\mathbf{9}, 3$ (2008) 205-230

9, 3 (2008) 299-316

9, 1 \& 2 (2008) 73-82

9, 4 (2008) 471-486

9, 4 (2008) 487-505

9, 1 \& 2 (2008) 1-29

9, 1 \& 2 (2008) 83-97

9, 1 \& 2 (2008) 83-97

9, 4 (2008) 425-438

9, 4 (2008) 351-367

9, 4 (2008) 389-408

9, 3 (2008) 299-316

9, 4 (2008) 389-408

9, 4 (2008) 409-423

$\mathbf{9}, 1$ \& 2 (2008) 127-139

9, 4 (2008) 487-505

9, 1 \& 2 (2008) 99-126

9, 3 (2008) 231-254

9, 4 (2008) 425-438

9, 1 \& 2 (2008) 53-72

9, 3 (2008) 255-276

9, 1 \& 2 (2008) 157-176

9, 4 (2008) 409-423

9, 4 (2008) 439-454

9, 1 \& $2(2008) 1-29$

$\mathbf{9}, 1$ \& 2 (2008) $73-82$

9, 1 \& 2 (2008) 1-29

9, 4 (2008) 455-470

9, 1 \& 2 (2008) 127-139

9, 4 (2008) 317-335 
Tan, J. J. M., see Lin, C.-K.

Taniar, D., see Zhao, G.

Tayu, S., Al-Mutairi, T. G. and Ueno, S., Cost-constrained minimum-delay multicasting

Ueno, S., see Tayu, S.

Valencia, D. S. and Scherson, I. D., Cost-performance analysis of service-address-routed least-common-ancestor networks

Vanderbauwhede, W., see Moadeli, M.

Wang, P., see Qu, Y.

Xuan, K., see Zhao, G.

Xuefen, C., see Jing, Z.

Yang, L. T., see Qu, Y.

Zhao, G., Xuan, K., Taniar, D. and Srinivasan, B., Incremental k-nearest-neighbor search on road networks
9, 1 \& $2(2008) 83-97$

$\mathbf{9}, 4(2008) 455-470$

9, 1 \& 2 (2008) $141-155$

$\mathbf{9}, 1 \& 2(2008) 141-155$

9, 1 \& 2 (2008) $157-176$

9, 4 (2008) 409-423

9, 4 (2008) 425-438

9, 4 (2008) 455-470

9, 4 (2008) 369-387

$\mathbf{9 , 4}(2008) 425-438$

$\mathbf{9}, 4(2008) 455-470$ 